Max-Planck-Institut für demografische Forschung

Max Planck Institute for Demographic Research

Doberaner Strasse $114 \cdot$ D-18057 Rostock · GERMANY

Tel +49 (0) 3812081 - 0; Fax +49 (0) 3812081 - 202;

http://www.demogr.mpg.de

MPIDR WORKING PAPER WP 2002-043

SEPTEMBER 2002

\title{
Post-Darwinian Longevity
}

James W. Vaupel (jwv@demogr.mpg.de)

(c) Copyright is held by the authors.

Working papers of the Max Planck Institute for Demographic Research receive only limited review.

Views or opinions expressed in working papers are attributable to the authors and do not necessarily reflect those of the Institute. 


\section{Post-Darwinian Longevity}

by

James W. Vaupel

Draft: 19 April 2002 with some revisions 11 September 2002

Forthcoming in a special issue of Population and Development Review

The force of evolution peters out with age. The age-specific power of natural selection depends on the proportion of individuals who survive and these individuals' remaining contribution to reproduction. Few individuals reach advanced ages. For species that do not continue to grow, fertility falls with age. In many social species the elderly contribute to reproduction by nurturing their younger relatives (Carey and Gruenfelder, 1997). The net contribution of the elderly, however, diminishes with decrepitude, and the dilution of their genes in successive generations weakens the action of natural selection.

Fisher (1930), Haldane (1941), Medawar (1946, 1952), Williams (1957), Hamilton (1966) and Charlesworth $(1994,2001)$ develop the notion that senescence results from the declining intensity with age of natural selection. Mutations that are harmful at older ages accumulate because there is only weak selection against such mutations. At post-reproductive ages there is no Darwinian culling to impede the spread of mutations that are lethal at those ages but have no effect (or a positive effect) at younger ages (Haldane, 1941; Charlesworth and Partridge, 1997; Partridge and Mangel, 1999). Hence, as Shripad Tuljapurkar critically remarked, there should be a black hole of mortality at the age when reproduction ceases.

Such a wall of death has not been observed in any species comprised of individuals that reproduce at more than one age. Indeed, death rates tend to decelerate with age in many species, and for some species death rates decline after some age (Vaupel, 1997; Vaupel et al. 1998). It is possible that late-acting deleterious mutations are rare or non-existent. If such mutations do exist, then Charlesworth (2001) concludes that they must have negative effects on fitness at younger ages and hence be quelled by natural selection.

Evolutionary geneticists have an impressive armamentarium of concepts and methods for thinking about mortality trajectories at reproductive ages. The research by Charlesworth and Partridge (1997), Partridge and Mangel (1999) and Charlesworth (2001), together with studies by Tuljapurkar (1997), Pletcher and Curtsinger (1998, 2000), Pletcher, Houle and Curtsinger (1998, 1999), Promislow and Tatar (1998) and others, suggest the vitality of recent efforts to explain why survival does not plummet to zero when reproduction ceases. 
This line of research, however, does not and probably cannot answer questions about mortality in the post-reproductive span of life.

In this article I attempt to venture into the reaches of age that are beyond the force of natural selection, to shed some light on what determines the duration of post-reproductive survival. The general approach I suggest may also prove useful in addressing related questions, such as the following. What determines the trajectory of mortality at postreproductive ages? Under what circumstances does mortality reach a plateau? Under what circumstances does mortality decline after some age? What determines the level of postreproductive mortality?

The post-reproductive ages are, to use James R. Carey's phrase, post-Darwinian, in the sense that there is no longer any age-specific pressure from natural selection. The health and vitality, however, of individuals of some species when they enter the post-reproductive period of life is determined by evolutionary forces operating at younger ages. An analogy helps explain the mix.

The speed and trajectory of a ball are governed by the pitcher's strength and skill up to the moment when the ball leaves the pitcher's hand. Thereafter, the ball's course is determined by the force of gravity acting on the momentum of the ball. Similarly, the course of life up until the end of reproduction is determined by evolutionary forces. After reproduction ceases the remaining trajectory of life is determined by forces of wear, tear and repair acting on the momentum produced by Darwinian forces operating earlier in life. Reliability engineers who study wear, tear and repair refer to the failure of equipment and use failure-time as a synonym for lifespan. Hence, I will employ the phrase the "force of failure" as a shorthand for the action of the various forces that determine the failure of complicated systems, including living organisms. The force of failure is analogous to the force of gravity in the metaphor about a ball's path. The force of gravity acting on the momentum of the ball determines how far the ball will travel. The force of failure acting on the post-Darwinian momentum of an organism determines how long the organism will survive.

The dynamics of the force of failure hinge on the design of a complicated system. At post-reproductive ages the design is set: evolution can no longer modify it. At younger ages, however, the powerful creativity of evolution produces miracles of innovative design. Thus at younger ages it may be reasonable to focus attention on evolutionary forces rather than on reliability-engineering considerations. Nonetheless, evolution has to operate under constraints dictated by physical and chemical laws and engineering principles. Furthermore, evolution works to maximize fitness rather than design reliability. As in design of equipment by 
humans, designs favored by natural selection usually economize on materials and allow imperfections and variation. A full explanation of the trajectory of mortality at ages before the end of reproduction may largely build on evolutionary theory, but it will be partially based on considerations of reliability engineering. Shiro Horiuchi's pathbreaking article in this volume demonstrates this. Earlier research on reliability analyses of the aging of living organisms includes contributions by Abernathy (1979) and Gavrilov and Gavrilova (2001); the Gavrilovs' references are helpful but their purported facts and findings are unreliable.

To summarize: evolutionary pressures shape the design of a species and largely determine trajectories of fertility and survival. At post-reproductive ages, the force of failure determines the pattern of mortality. The force of natural selection peters out with age and the force of failure becomes more and more significant. The force of failure acts on postDarwinian momentum, i.e., on the legacy left by evolutionary pressures when the agespecific intensity of these pressures falls to negligible levels.

My thesis is that the force of natural selection governs the length of life of most individuals of a species in some environment, but that the force of failure governs the length of the outer tail of longevity. The start and end of the tail can be quantified in many ways. Here I use a simple expedient. I assume that the tail of longevity starts at the age when only ten percent of a cohort are still alive. For the species for which data on sizeable populations are available, reproduction at this age is negligible. Although this is a very rough indicator, I will consider this age to be the start of the post-Darwinian span of life. I assume that the tail of longevity ends at the maximum lifespan attained by the cohort being studied. The length of the tail of longevity is given by record lifespan minus the top tenth percentile of lifespan. The relative length of the tail is the length divided by this percentile.

\section{The Elusive Concept of Lifespan}

The lifespan of an individual is the duration of that individual's life. When, however, does life start? For humans, lifespans are usually measured from birth. If they were measured from conception and if 9 of 10 conceptions end in miscarriage, then human life expectancy would be cut by a factor of ten. The lifespan of other species is often measured from the start of some stage of life, e.g., emergence from the pupa for some insects.

For some species, age at death, especially in the wild, can be difficult to determine. Furthermore, individuals of some species spend long periods in hibernation or some other stage in which the flame of life barely flickers. 
Environmental conditions can drastically influence the duration of life for a species. Human life expectancy in some populations today is well under 50 and in some historical populations it was under 25, whereas for Japanese women today it is 85 (Oeppen and Vaupel, 2002; Japanese Ministry of Health, Labor and Welfare, 2002). Depending on diet and other conditions, the lifespan of medflies can be substantially extended (Carey et al., 1998). Queen bees can live an order of magnitude longer than workers, even though queens and workers are genetically identical (Finch 1990). Point mutations of one or two genes can double or triple lifespans (Johnson 1997).

These well-known complexities of the concept of lifespan imply that lifespan data must be treated with caution, especially when individuals from very different species or in very different environments are compared. Here I would like to highlight an additional difficulty that is less well understood. Record lifespan, which is usually the only lifespan measure available for a species (Carey and Judge 2000), can vary considerably depending on population size and the trajectory of mortality at advanced ages. This trajectory, as I argued above, is determined by the force of failure operating on post-Darwinian momentum. It is often assumed that the average duration of life for individuals in a species is some more-orless fixed fraction of the maximum lifespan observed for individuals in the species. If this were true, then record lifespan could be used as a surrogate for average lifespan. The facts, however, are very different.

It used to be believed that for most species death rates tend to increase exponentially with age at adult ages. It is now known that there is a deceleration of the increase in death rates at older ages and sometimes a decline after some age. For humans, a simple exponential curve (the Gompertz curve) fits mortality data for most populations serviceably well from age 35 or so up to age 95 or so. After age 95, a marked deceleration of the rate of mortality increase is observed in populations with reliable data (Thatcher et al. 1998). After age 110, human mortality may reach a plateau or even start to fall (Vaupel et al. 1998; Robine and Vaupel, 2002). For other species for which large populations have been followed from birth to death, deceleration of the rise of mortality is also observed, usually with a plateau that is reached when a few individuals are still alive and sometimes with a strong decline in mortality after some age (Vaupel, 1997; Vaupel et al., 1998).

It is useful, then, to consider three cases: (1) death rates increase exponentially with age; (2) death rates reach a plateau; and (3) death rates decline with age after some age. For simplicity, assume that only ten percent of the population is alive at the age when death rates reach ten percent per unit of time (year, month, week or day depending on the species). In 
Table 1, three specific formulas are used to model mortality after this post-Darwinian age. In the first model, death rates rise exponentially. In the second model, death rates remain at ten percent. And in the third model, death rates gradually fall from ten percent to one percent. In the table, four different population sizes are considered-populations with ten observations of old-age lifespan, populations with a thousand observations, populations with a million observations, and populations with a billion observations.

Note that if mortality increases exponentially, then the post-Darwinian span of life is only moderately influenced by population size. If, however, mortality declines, then the postDarwinian tail lengthens dramatically with increases in population size. Also note that at any given population size, the duration of life from the onset of old age to the longevity record is very long for species for which mortality declines with age and quite long for species for which mortality has leveled off.

Table 2 provides some empirical data about tails of longevity for several species. In modern human populations with low levels of mortality, the tail of longevity is relatively short. As indicated in Table 2, it used to be longer. For genetically-identical lines of rats, Drosophila, and nematode worms, even longer tails are observed. For one-celled yeast and for large, genetically-heterogeneous populations of invertebrates, the tails are very long.

Caution must be used in interpreting the table because population sizes vary greatly. As shown in Table 1, population size can have a substantial impact on maximum lifespan, especially when mortality levels off or declines. Furthermore, regardless of population size, the trajectory of mortality at advanced ages has a major impact on record lifespan. For humans (at least in modern populations for which reliable data are available), mortality increases more or less exponentially until age 95 or so and does not level off until age 110 (Thatcher et al., 1998; Robine and Vaupel, 2002). For the genetically-identical populations of flies and worms in Table 2, a mortality plateau is reached relatively earlier in life (see Shiro Horiuchi's article in this volume). Yeast mortality fluctuates, rising and falling and rising again (Vaupel et al. 1998). For the genetically-heterogeneous populations of insects in Table 2, mortality falls sharply at older ages (Vaupel et al. 1998). The length of the tails in Table 2 is thus consistent with the general thrust of Table 1.

In any case, the tails of longevity in Table 2 are astounding. For Japanese women today, the maximum span of life is a mere 16 percent higher than the top tenth percentile. For yeast, medflies and parasitoid wasps, however, maximum lifespan is more than triple the advanced old age that only a tenth of the population attains. So long for some invertebrates and so short for modern humans, this is a tail on which to hang a tale—of reliability. 
Determinants of the Length of the Tail of Longevity

Using some simple models, I have started to explore the impact of various design features and environmental characteristics on the remarkably dissimilar gaps between record lifespan and the top tenth percentile of lifespan. So far I have looked at three aspects of a species' physical design—repair, redundancy, and individual variability. In addition, I have begun to consider the impact of environmental variability.

Consider first an organism that suffers some constant hazard of death $\mu$ at all ages. The chance of survival to age $\mathrm{x}$ is then given by $s(x)=\exp (-\mu x)$. Hence the age at which only ten percent of the population is still alive, $x_{.10}$, is given by $.1=\exp \left(-\mu x_{.10}\right)$, which implies $x_{.10}=-\ln (.1) / \mu$. In a cohort of $n$ individuals, the chance that all are dead by age $\mathrm{x}$ is $(1-\exp (-\mu x))^{n}$. Therefore $x^{*}$, the median of the maximum lifespan, is given by $.5=\left(1-\exp \left(-\mu x^{*}\right)\right)^{n}$, which implies $x^{*}=-\ln \left(1-.5^{1 / n}\right) / \mu$. The relative length of the tail of longevity, $\tau$, can thus be calculated by $\tau=\frac{\ln \left(1-.5^{1 / n}\right)}{\ln (.1)}-1$. Note that $\mu$, the force of mortality, drops out of this formula. If the hazard of death is five percent, then ten percent of the population can be expected to survive to age 46. If the cohort has 1000 members, then the median age at which the last individual dies is 145.5 . The tail of longevity is 2.16 times longer than the age to which only a tenth of the population survive. (The life expectancy of this species, by the way, is just the inverse of $\mu$, or 20 when $\mu$ is five percent. If $\mu$ were one percent, then life expectancy would be 100 . The relative length of the longevity tail, however, would be 2.16 regardless of the constant value of $\mu$.)

Consider now another species that has two "systems" such that death results only when both systems fail. As a simple analogy, consider human eyesight. Blindness occurs if both eyes fail. How does such redundancy affect the longevity tail? The age to which ten percent of the population survive is given by $.1=1-\left(1-\exp \left(-\mu x_{.10}\right)\right)^{2}$, so $x_{.10}=-\ln \left(1-.9^{1 / 2}\right) / \mu$. The median of the maximum lifespan achieved is given by $.5=\left(\left(1-\exp \left(-\mu x^{*}\right)\right)^{2}\right)^{n}$, so $x^{*}=-\ln \left(1-.5^{1 / 2 n}\right) / \mu$. Thus the relative length of the tail is given by $\tau=\frac{\ln \left(1-.5^{1 / 2 n}\right)}{\ln \left(1-.9^{1 / 2}\right)}-1$. Note again that $\mu$ drops out of the formula for the tail. Hence the force of mortality for the redundant species could be set so that the life expectancy (or any other index of survival) for this species was the same as for the simpler species: $\tau$ will 
not be affected. In a population of size 1000 with $\mu$ equal to 5 percent, the relative length of the tail of longevity is 1.68 for the redundant species compared with 2.16 for the simpler species.

This finding — redundancy reduces the relative length of the tail of longevity-holds for all the species designs I have explored. It is easily shown that putting more and more systems in parallel, such that all have to fail before the organism dies, reduces $\tau$. I have also looked at somewhat more complicated designs, with both parallel and serial elements, using computer simulation to estimate $\tau$. It may be possible to prove the result under general conditions, perhaps using the theory of phase-type distributions, but so far I have only considered some simple systems with independent elements that suffer constant mortality.

I have also explored the impact of allowing repair. When an individual is about to die, the individual can be granted a second chance. Or individuals can be given a new lease on life with some probability. Individuals could be given nine chances or more, with some probabilities. "Repaired" or "resuscitated" individuals could have the same or worse survival chances than they faced before. Anatoli Yashin and I explored such models in the context of human life expectancy (Vaupel and Yashin 1986, 1987) and some of our results could be extended to analyses of longevity tails. My preliminary mathematical and computersimulation results suggest that the more repair allowed, the shorter the relative tail of longevity. This may be a result that holds under general or fairly general conditions.

Instead of assuming that all individuals face the same mortality schedule, individual variability could be allowed. One way to model such heterogeneity is the frailty model introduced by Vaupel, Manton and Stallard (1979). If frailty is assumed to be gamma distributed with mean one and variance $\sigma^{2}$, then the chance of surviving to age $x$ is given by $s(x)=\left(1-\sigma^{2} H(x)\right)^{-1 / \sigma^{2}}$, where $H(x)$ is the cumulative hazard for an individual of frailty one. If the force of mortality follows a Gompertz curve, $\mu(x)=a \exp (b x)$, then $H(x)=\left(a \sigma^{2} / b\right)(\exp (b x)-1)$. The age to which a tenth of the population survive is given by $s\left(x_{.10}\right)=.1$ and the median of the maximum age attained is given by $\left(\left(1-s\left(x^{*}\right)\right)^{n}=.5\right.$. It is possible to solve for $x_{.10}$ and $x^{*}$ in terms of the parameters $a, b$, and $\sigma^{2}$ and then to compute the derivative of $\tau$ with respect to $\sigma^{2}$. This derivative is always positive, which implies that the greater the heterogeneity in individual frailty, the longer the relative length of the tail of longevity. This result may also hold for trajectories of mortality other than the Gompertz curve, for distributions of frailty other than the gamma distribution, and for more 
complicated models of individual variability than the frailty model. Computer-simulation experiments I have done suggest that the result may hold under a wide range of plausible conditions.

Shiro Horiuchi, in this volume, estimates values for $\sigma$ in models with gammadistributed frailty. He finds that individual variability is lowest for humans, higher in genetically-identical lines of Drosophila and nematode worms, and highest in geneticallyheterogeneous populations of medflies, parasitoid wasps, and bean beetles. This ordering is consistent with the values of $\tau$, the relative length of the tail of longevity, presented above in Table 2.

Finally, I have explored the impact of environmental variability on the longevity tail. I did this by allowing the level of mortality to stochastically vary from one time period to the next, in simple computer-simulation models. The greater the environmental variability, the longer the relative length of the tail of longevity. Often in my experiments, however, the effect was small, especially when mortality was allowed to randomly jump to some level above or below its mean level from one short time interval to the next. The mortality fluctuations tended to cancel each other out by the time older ages were reached, so that the age at which a tenth of the population survives and the highest age attained were not much affected.

To summarize, the less redundancy, the less repair, the more individual variability, and the more environmental variability, the longer the relative length of the tail of longevity. These results are preliminary, but suggestive. They seem consistent—or at least not inconsistent-with the empirical results in Table 2.

The combination of redundancy, repair, and low variability among individuals might be referred to as the "reliability" of a species. Humans are a reliable species in a steady environment; medflies are an unreliable species in an uncertain environment. Shiro Horiuchi, in his article in this volume, rightly emphasizes the "quality-control" of individual variability. Redundancy and repair are also important. Humans have more design redundancy than worms and worms have more that single-celled yeast. The insects and worms in Table 2 are post-mitotic: they cannot replace cells and hence have limited repair capabilities.

The basic finding of this article is that reliable species have short tails of longevity and unreliable species have long tails of longevity. Because this may seem counter-intuitive or even paradoxical, it is worth careful consideration. If the tail of longevity is short relative to a species' average lifespan, then mortality at advanced ages is high compared with mortality at younger ages. That is, death rates before old age are relatively low. Hence, the 
fundamental result of this article also can be expressed as follows: reliable species enjoy low death rates at younger ages and relatively high death rates at older ages. In unreliable species, the gap between mortality at advanced ages vs. younger ages is smaller and sometimes even negative.

Note that in principle a unreliable species could have low mortality and long life expectancy. Consider, for instance, a system consisting of a single element with no repair. If the force of mortality for this element were low, life expectancy would be long-and the relative tail of longevity would be very long, as indicated in the first example above. So, an unreliable species is not necessarily the same as a low-quality species. In most cases, however, unreliable species suffer high death rates. Also note that an uncertain environment could, on average, be a favorable one.

\section{Conclusion}

In addition to mobilizing the concepts and methods of evolutionary thinking to try to address the black-hole problem of the theories of mutation accumulation and antagonistic pleiotropy, researchers can address a stimulating new question. What degree of reliability maximizes Darwinian fitness in different environments and ecological niches? A species' reliability is determined by natural selection operating during the reproductive period of life. A species' reliability, interacting with the uncertainty of the environment, determines the length of the post-reproductive lifespan. The biology of longevity has to be considered in the light of evolution, but it also has to be considered in the light of reliability engineering. And both evolution and reliability engineering have to be considered in the light of population thinking, i.e., demography.

Hence, demographic perspectives on the comparative biology of longevity can produce illuminating insights that augment the research of evolutionary biologists. This article and the other articles in this volume provide examples. Evolutionary biologists have devoted some attention to post-reproductive life (Wachter and Finch 1997), but much more research is warranted. The trajectory of mortality at advanced ages is of fundamental scientific interest to researchers interested in aging. The dramatic rise of human life expectancy and the rapid aging of human populations make understanding the outer reaches of survival highly relevant. Following Lotka's lead, demographers can continue to make substantial contributions to knowledge about the forces that govern life. 
TABLE 1: Median Record Lifespan and 98\% Range of Record Lifespans for Small and Large Cohorts and for Increasing, Level, and Declining Age-Specific Mortality.

\begin{tabular}{cccc}
$\begin{array}{c}\text { Population } \\
\text { Size }\end{array}$ & $\begin{array}{c}\text { Increasing } \\
\text { Mortality }\end{array}$ & $\begin{array}{c}\text { Constant } \\
\text { Mortality }\end{array}$ & $\begin{array}{c}\text { Declining } \\
\text { Mortality }\end{array}$ \\
\hline 10 & 13 & 27 & 180 \\
7 to 21 & 10 to 69 & 21 to 600 \\
1000 & 21 & 73 & 637 \\
19 to 25 & 54 to 115 & 394 to 1061 \\
$1,000,000$ & 27 & & 142 \\
& 26 to 30 & 123 to 184 & 1139 to 1752 \\
$1,000,000,000$ & 31 & & 211 \\
& 29 to 33 & 192 to 253 & 1728 to 2443
\end{tabular}

Source: Author's calculations. The record lifespans are computed from the age when the force of mortality reached 10 percent. From this starting age, the trajectory of increasing mortality is given by $\mu(x)=.1 \exp (.1 x)$. In the constant mortality case, the force of mortality was held at ten percent per unit of time. The trajectory of decreasing mortality is $\mu(x)=.09 \exp (-.1 x)+.01$. In a cohort of $n$ individuals, the probability that all are dead by age $x$ is $(1-s(x))^{n}$, where $s(x)=\exp \left(-\int_{0}^{x} \mu(a) d a\right.$ is the probability of surviving from the starting age to age $x$. Hence the $p$ th fractile of the distribution of maximum lifespan can be calculated as the age such that $s(x)=1-p^{1 / n}$. The medians in the table were computed by setting $p=0.5$; for the range, values of $p=0.01$ and $p=0.99$ were used. 
TABLE 2: The Relative Length of the Tail of Longevity for Several Species.

\begin{tabular}{lcccc} 
& Size of & Age when 10\% & Maximum age & $\begin{array}{l}\text { Relative length of } \\
\text { the tail of longevity, }\end{array}$ \\
Population & cohort & survive, $x_{.10}$ & attained, $x_{\max }$ & $\tau=\left(x_{\max }-x_{.10}\right) / x_{.10}$ \\
\hline
\end{tabular}

\section{Humans:}

Japanese females

1999 period lifetable $\approx 10^{6}$

97 years

113

0.16

Swedish females, cohort of 1870

$60,000 \quad 86$

110

0.28

Swedish females,

Cohort of 1760

30,000

77

102

0.32

Hungary, both

sexes, 0-399 AD

$184 \quad 62$

100

0.61

Genetically-identical:

Rats, both sexes

$770 \quad 698$ days

1427

1.04

Drosophila

$16,000 \quad 52$

95

0.83

Nematode worms,

Wild-type

20,000

19

32

0.68

Nematode worms,

Age-1 mutant

$10,000 \quad 27$

54

1.00

Yeast (S88C)

$$
\approx 10^{6}
$$

119

Yeast (EG103)

$$
\approx 10^{6}
$$

10

67

5.90

Genetically-heterogeneous:

Medflies, both sexes $1,200,000$

Anastrepha ludens,

both sexes

$$
1,600,000 \quad 50
$$

Anestrepha serpentina,

both sexes

$350,000 \quad 33$

90

Anestrepha obliqua,

both sexes

$300,000 \quad 32$

75

1.34

Parasitoid wasps,

both sexes

30,000

17

70

3.12 
Notes: Japanese and Swedish data are from the Human Mortality Database maintained by the University of California, Berkeley, and the Max Planck Institute for Demographic Research (http://www.demogr.mpg.de/databases ). The data for Hungary are from a lifetable, based on gravestone epitaphs, estimated by Acsadi and Nemeskeri (1970); these data may not be reliable. In particular, it is unlikely that the maximum age actually was 100 (Jeune and Vaupel 1995, 1999). It is more likely that it was 85 or 90 which would give a value of $\tau$ of 0.37 or 0.45 . Numerous lifetables based on age estimation of skeletal remains have been published, but the methods are highly questionable (Hoppa and Vaupel, 2002). The nonhuman data are all from the Nonhuman Mortality Database maintained at the Max Planck Institute for Demographic Research. The rat data were supplied by Vladimir Anisimov; they pertain to a Wistar-derived line called LIO. The Drosophila melanogaster data are from James W. Curtsinger, the nematode worm (C. elegans) data are from Thomas E. Johnson, and the yeast (S. cerevisiae) data are from Nadège Minois at the Max Planck Institute for Demographic Research. The medfly (Ceratitis capitata), Anastrepha, and parasitoid wasp (Diachasmimorpha longiacaudtis) statistics are from experiments conducted in Tapachula, Mexico, under the direction of James. R. Carey. Note that $x_{\max }$ is the empirical maximum age attained and hence is different from the theoretical $x^{*}$. Hence $\tau$ in this table is subtly different from the $\tau$ that is a function of $x^{*}$. 


\section{References}

Abernathy, J.D. (1979) “The Exponential Increase in Mortality Rate with Age Attributed to Wearing-Out of Biological Components”, J. Theoretical Biology 80: 333-354.

Acsadi, G. and J. Nemesceri (1970) History of Human Lifespan and Mortality. Budapest: Akademiai Kiado.

Carey, J.R. and C. Gruenfelder (1997) "Population Biology of the Elderly" in K.W. Wachter and C.E. Finch (eds.) Between Zeus and the Salmon. Washington, D.C.: National Academy Press, pp. 127-160.

Carey, J.R. and D. S. Judge (2000) Longevity Records: Life Spans of Mammals, Birds, Amphibians, Reptiles, and Fish. Odense, Denmark: Odense U. Press.

Carey, J.R., P. Liedo, H.-G. Müller, J.-L. Wang, and J.W. Vaupel (1998) "Dual Modes of Aging in Mediterranean Fruit Fly Females" Science 281: 996-998.

Charlesworth, B. (1994) Evolution in Age-Structured Populations. Cambridge: Cambridge U. Press.

Charlesworth, B. and L. Partridge (1997) "Levelling of the Grim Reaper", Current Biology 7: 440-442.

Finch, C.E. (1990) Longevity, Senescence, and the Genome. Chicago: University of Chicago Press.

Fisher, R.A. (1930) The Genetical Theory of Natural Selection. Oxford: Oxford U. Press.

Gavrilov, L.A. and N.S. Gavrilova (2001) "The Reliability Theory of Aging and Longevity", J. Theoretical Biology 213: 527-545.

Haldane, J.B.S. (1941) New Paths in Genetics. London: Allen and Unwin.

Hamilton, W.D. (1966) "The Moulding of Senescence by Natural Selection”, J. Theoretical Biology 12: 12-45.

Hoppa, R.D. and J.W. Vaupel (2002) Paleodemography: Age Distributions from Skeletal Samples. Cambridge, England: Cambridge U. Press.

Japanese Ministry of Health, Labor and Welfare (2002)

http://www.mhlw.go.jp/english/database/db-hw/lifetb00/part1.html .

Jeune, B. and J.W. Vaupel, eds. (1995) Exceptional Longevity: From Prehistory to the Present. Odense, Denmark: Odense U. Press.

Jeune, B. and J.W. Vaupel, eds. (1999) Validation of Exceptional Longevity.. Odense, Denmark: Odense U. Press.

Johnson, T.E. (1997) “Genetic Influences on Aging”, Experimental Gerontology 12: 11-21. 
Medawar, P.B. (1946) “Old Age and Natural Death” Modern Quarterly 1: 30-56.

Medawar, P.B. (1952) An Unsolved Problem in Biology. London: H.K. Lewis.

Oeppen, J. and J.W. Vaupel (2002) "Broken Limits to Life Expectancy", Science 296(5570): 1029-31. Available online at http://www.demogr.mpg.de/publications/files/brokenlimits.htm

Partridge, L. and M. Mangel (1999) "Messages from Mortality: The Evolution of Death Rates in the Old", Trends in Ecology and Evolution 14: 438-442.

Pletcher, S.D. and J.W. Curtsinger (1998) "Mortality Plateaus and the Evolution of Senescence: Why Are Old-Age Mortality Rates So Low?”, Evolution 52: 454-464.

Pletcher, S.D. and J.W. Curtsinger (2000) "The Influence of Environmentally Induced Heterogeneity on Age-Specific Variance for Mortality Rates" Genetic Research 75: 321-329.

Pletcher, S.D., D. Houle and J.W. Curtsinger (1998) "Age-Specific Properties of Spontaneous Mutations Affecting Mortality in Drosophila melanogaster" Genetics 148: 287-303.

Pletcher, S.D., D. Houle and J.W. Curtsinger (1999) "The Evolution of Age-Specific Mortality Rates in Drosophila melanogaster among Unselected Lines" Genetics 153: 813823.

Promislow, D.E.L. and M. Tatar (1998) "Mutation and Senescence: Where Genetics and Demography Meet” Genetica 102/103: 299-313.

Robine, J.-M. and J.W. Vaupel (2002) "Emergence of Supercentenarians in Low Mortality Countries", North American Actuarial Journal forthcoming.

Thatcher, A.R., V. Kannisto and J.W. Vaupel (1998) The Force of Mortality at Ages 80 to 120. Odense, Denmark: Odense U. Press. Available online at www.demogr.mpg.de .

Tuljapurkar, S. (1997) "The Evolution of Senescence” in K.W. Wachter and C.E. Finch (eds.) Between Zeus and the Salmon. Washington, D.C.: National Academy Press, pp. 65-77.

Vaupel, J.W. (1997) "Trajectories of Mortality at Advanced Ages" in K.W. Wachter and C.E. Finch (eds.) Between Zeus and the Salmon. Washington, D.C.: National Academy Press, pp. $17-37$.

Vaupel, J.W., J.R. Carey, K. Christensen, T.E. Johnson, A.I. Yashin, N.V. Holm, I.A. Iachine, V. Kannisto, A.A. Khazaeli, P. Liedo, V.D. Longo, Y. Zeng, K.G. Manton, and J.W. Curtsinger (1998) "Biodemographic Trajectories of Longevity”, Science 280: 855-860.

Vaupel, J.W., K.G. Manton, E. Stallard (1979) "The Impact of Heterogeneity in Individual Frailty on the Dynamics of Mortality", Demography 16: 439-454.

Vaupel, J.W. and A.I. Yashin (1986) "Targeting Lifesaving: Demographic Linkages between Population Structure and Life Expectancy”, European Journal of Population 2: 335-360. 
Vaupel, J.W. and A.I. Yashin (1987) "Repeated Resuscitation: How Lifesaving Alters Life Tables", Demography 24: 123-135.

Wachter, K.W. and C.E. Finch (eds.) Between Zeus and the Salmon. Washington, D.C.: National Academy Press.

Williams, G.C. (1957) "Pleiotropy, Natural Selection and the Evolution of Senescence", Evolution 11: 398-411. 


\section{Acknowledgments}

The author thanks Annette Baudisch, James R. Carey, Maxim Finkelstein, Jutta Gampe and Shiro Horiuchi for helpful comments.

\section{The Author}

James W. Vaupel is Founding Director of the Max Planck Institute for Demographic Research, Rostock, Germany. 